\title{
Walking toward a convergence in aging research
}

\author{
Mark A. Smith ${ }^{1 *}$ and Gemma Casadesus ${ }^{2 *}$ \\ 1 Department of Pathology, Case Western Reserve University, Cleveland, OH, USA \\ 2 Department of Neurosciences, Case Western Reserve University, Cleveland, OH, USA \\ *Correspondence: *mark.smith@case.edu; **"gxc40@case.edu
}

Men ought to know that from nothing else but the brain come joys, delights, laughter and sports, and sorrows, griefs, despondency, and lamentations... And by the same organ we become mad and delirious, and fears and terrors assail us, some by night, and some by day, and dreams and untimely wanderings, and cares that are not suitable, and ignorance of present circumstances, desuetude, and unskilfulness. All these things we endure from the brain, when it is not healthy...

-Hippocrates, On the Sacred Disease

The brain is responsible for providing everything from the basic involuntary physiological events that allow one to breathe and live, to the conscious actions and thoughts that dictate the very essence of mankind. As such, the preservation of brain function as individuals age is not only crucial to support the ability to live but also the ability to maintain their individuality. Aging is an inevitable process that everyone faces and one that has captivated people's minds since the beginning of our existence. Promises of life extension and immortality have been marketed from alchemists to charlatans and continue to flood popular media even today. The apparent desire to beat the ravages of age often outweighs rigorous thought, and that very popular obsession has sometimes diluted the seriousness of the field. That said, understanding the aging nervous system is a field that has attracted some of the brightest and best clinicians as well as basic scientists whose intellectual ideas and scholarly research bear on the field in an effort to reach an understanding of the fundamental mechanistic events underlying aging.

Healthy lifestyles, medical advances in the 20th century, and the coming of age of baby boomers have brought out the reality of living longer, and uncovered the challenges that an aging population brings to human lives, health systems, and communities. In the 21 st century, the number of individuals reaching 65 years of age is increasing exponentially. The number has increased 20 -fold in the last century such that the spectrum of diseases that affect the population has changed and "aging" itself has become a prevalent condition. Understanding the causes of such age-related problems and the ways in which these problems can be most effectively treated or prevented is a challenge faced by us as a society, a challenge demanding to be addressed in a scientifically rigorous fashion. Over time, numerous theories on aging have been proposed. However, the fundamental link that unites these theories toward a cohesive understanding of aging is still lacking. The dissection of genetics versus environment and the way in which the genetics and the envi- ronment interact to affect development and maturation and demise is a pending subject for the research community. Pressing questions that need to be addressed are those concerning mechanisms associated with brain aging vulnerability, and the way in which selective neuronal populations become susceptible to disease as people age. Also, what makes certain individuals maintain incredible mental vitality and lucidity throughout life, and why others lose the essence of their individuality is key to understanding the fundamental layout of not only aging, but also the brain itself. Unfortunately, understanding normal healthy brain function is in its infancy, and while advances in all research disciplines spanning areas as diverse as studying aging genetics in invertebrate or vertebrate models such as zebrafish, or functional neuroimaging in the human aged brain are promising, more often than not understanding the complexity of brain physiology is diminished by reductionist approaches. Such crudeness is only exacerbated by attempts to understand abnormal physiology and pathology.

To date, aging neurosciences research is divided across disciplines. The study of age-related molecular switches driving cellular processes from stem cells to mature neurons is, to some extent, studied separately from how the switches are genetically determined, or the process in which environmental factors such as stress, sex-specific events such as menopause, nutrition or even climate affect the blueprints. In addition, the functional manifestations of aging encompassing cognitive, motor, and emotional age-related changes are often studied as separate entities. The challenge, therefore, is to unite the fields into closer approximation to allow a constructive input and open synergetic dialogue between these closely related research entities.

Last scene of all that ends this strange, eventful history, is second childishness and mere oblivion. I am sans teeth, sans eyes, sans taste, sans everything.

-William Shakespeare, As You Like It

Received: 26 February 2009; published: 01 May 2009

Citation: Front. Neurosci. (2009) 3,1: 1. doi: 10.3389/neuro.01.012.2009

Copyright (c) 2009 Smith and Casadesus. This is an open-access publication subject to an exclusive license agreement between the authors and the Frontiers Research Foundation, which permits unrestricted use, distribution, and reproduction in any medium, provided the original authors and source are credited. 